http://jmscr.igmpublication.org/home/ ISSN (e)-2347-176x ISSN (p) 2455-0450

crossref DOI: https://dx.doi.org/10.18535/jmscr/v8i1.11

\title{
CT Angiography detection of ruptured intracranial aneurysms with subarachnoid hemorrhage in a hospital-based population
}

\author{
Authors
}

\author{
Dr Sumana Bingi ${ }^{1 \%}$, Dr Venkatesh Basina ${ }^{1}$, Dr N Giridhar Gopal ${ }^{2}$ \\ ${ }^{1}$ Postgraduate, Department of Radiodiagnosis, GEMS \\ ${ }^{2}$ Professor, Department of Radiodiagnosis, GEMS \\ *Corresponding Author \\ Dr Sumana Bingi \\ Postgraduate, Department of Radiodiagnosis, GEMS
}

\begin{abstract}
Background: Rupture of an intracranial aneurysm presents as subarachnoid hemorrhage (SAH). SAH is a catastrophic event with an overall mortality rate varying from 32-37\%. It may be commonly be associated with intraparenchymal hemorrhage (IPH), intraventricular hemorrhage (IVH), subdural hemorrhage (SDH).

Objectives: To know the frequency, most common site of aneurysms and associated anatomical variants in patients with ruptured intracranial aneurysms.

Material and Methods: The study includes 150 patients who have undergone CT Angiography at with symptoms like headache, neck stiffness, and clinically diagnosed SAH. The required data were collected on a retrospective basis from the database available at Great eastern medical school and hospital from January 2017 to March 2019. From the obtained data, the prevalence, common site of intracranial aneurysms and anatomical variants (if any) were evaluated.

Results: A total of 150 patients have undergone non-contrast CT and further angiographic imaging at Great eastern medical school and hospital from January 2017 to March 2019, of which 20 patients were documented with ruptured intracranial aneurysms. Of these patients, the mean age was 51 years with equal incidence in males and females. $65 \%$ of the aneurysms were seen on the left and $35 \%$ on the right side. $84 \%$ of the aneurysms were in the anterior circulation and $16 \%$ in the posterior circulation. Isolated aneurysms were seen in $70 \%$ and synchronous aneurysms in $30 \%$ of the study population. The most common site of aneurysms was in the supraclinoid ICA corresponding to $34.6 \%$. The other sites were $30.7 \%$ in MCA, $11.5 \%$ in Acom, $11.5 \%$ in PCA, 3.8\% in Pcom, 3.8\% in ACA and 3.8\% in Vertebral arteries respectively. Anatomical variants were seen with $25 \%$ of the study population, predominantly in the posterior circulation.

Conclusion: Any CT scan showing an intraparenchymal hemorrhage in the temporal lobe, with or without a frontal, parietal and intraventricular extension should be looked in for any ruptured aneurysms even in the absence of SAH.

Keywords: Subarachnoid hemorrhage, Aneurysm, CT Angiography.
\end{abstract}

\section{Introduction}

Rupture of an intracranial aneurysm presents as subarachnoid hemorrhage (SAH) due to the flow of blood into the subarachnoid space. The variations in the major intracranial vasculature increase the hemodynamic stress and lead to 
developing an aneurysm, arteriovenous malformation, or cerebrovascular disease. SAH is a catastrophic event with an overall mortality rate varying from $32-37 \%$. It may be commonly be associated with intraparenchymal hemorrhage (IPH), intraventricular hemorrhage (IVH), subdural hemorrhage (SDH) in up to 34,17 , and $35 \%$ cases, respectively ${ }^{1}$.

\section{Material and Methods}

The study includes 150 patients who have undergone CT Angiography at GEMS as directed by the clinician presenting with symptoms like headache, neck stiffness, and clinically diagnosed $\mathrm{SAH}$. The required data were collected on a retrospective basis from the database available at Great eastern medical school and hospital from January 2017 to March 2019. The database was searched for patients with ruptured intracranial aneurysms, intraventricular and subarachnoid hemorrhages on non-contrast $\mathrm{CT}$ and $\mathrm{CT}$ angiography imaging. Those patients with angiographically documented aneurysms were reviewed with non-contrast $\mathrm{CT}$ and $\mathrm{CT}$ angiography images. From the obtained data, the prevalence, common site of intracranial aneurysms and anatomical variants (if any) were evaluated.

\section{Results}

There were a total of 150 patients who have undergone non-contrast CT and further angiographic imaging at Great eastern medical school and hospital from January 2017 to March 2019 , of which 20 patients were documented with ruptured intracranial aneurysms. Of these patients, the mean age was 51 years with equal incidence in males and females. $65 \%$ of the aneurysms were seen on the left and $35 \%$ on the right side. $84 \%$ of the aneurysms were in the anterior circulation and $16 \%$ in the posterior circulation (Figure 1). Isolated aneurysms were seen in $70 \%$ and synchronous aneurysms in $30 \%$ of the study population. The most common site of aneurysms was in the supraclinoid ICA corresponding to
$34.6 \%$. The other sites were $30.7 \%$ in MCA, 11.5 $\%$ in Acom, $11.5 \%$ in PCA, $3.8 \%$ in Pcom, $3.8 \%$ in ACA and $3.8 \%$ in Vertebral arteries respectively (Figure 2). Anatomical variants were seen with $25 \%$ of the study population, predominantly in the posterior circulation.

PERCENTAGE OF ANEURYSMS IN ANTERIOR AND POSTERIOR CIRCULATION

a ANTERIOR CIRCULATION $=$ POSTERIOR CIRCULATION

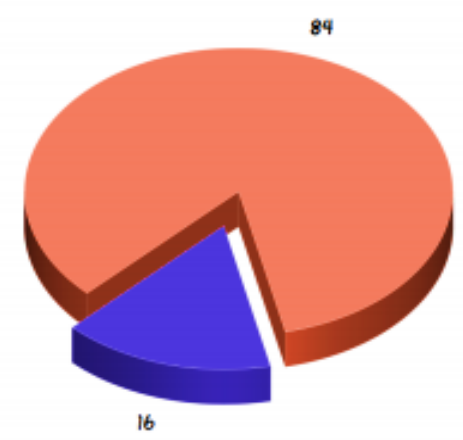

Fig.1: Percentage of aneurysms in the anterior and posterior circulation.

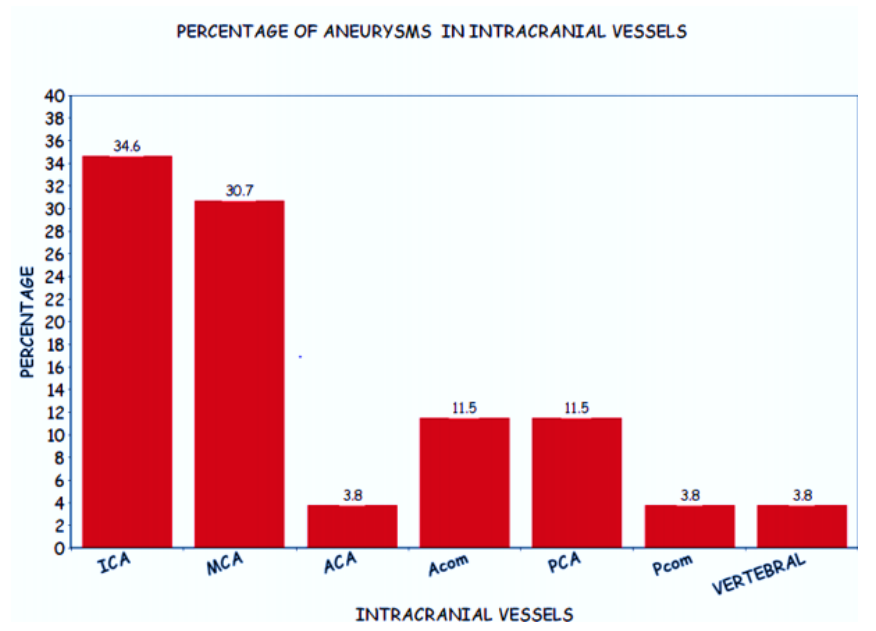

Fig.2: Percentage of aneurysms in intracranial circulation. 


\section{JMSCR Vol||08||Issue ||01||Page 91-96||January}

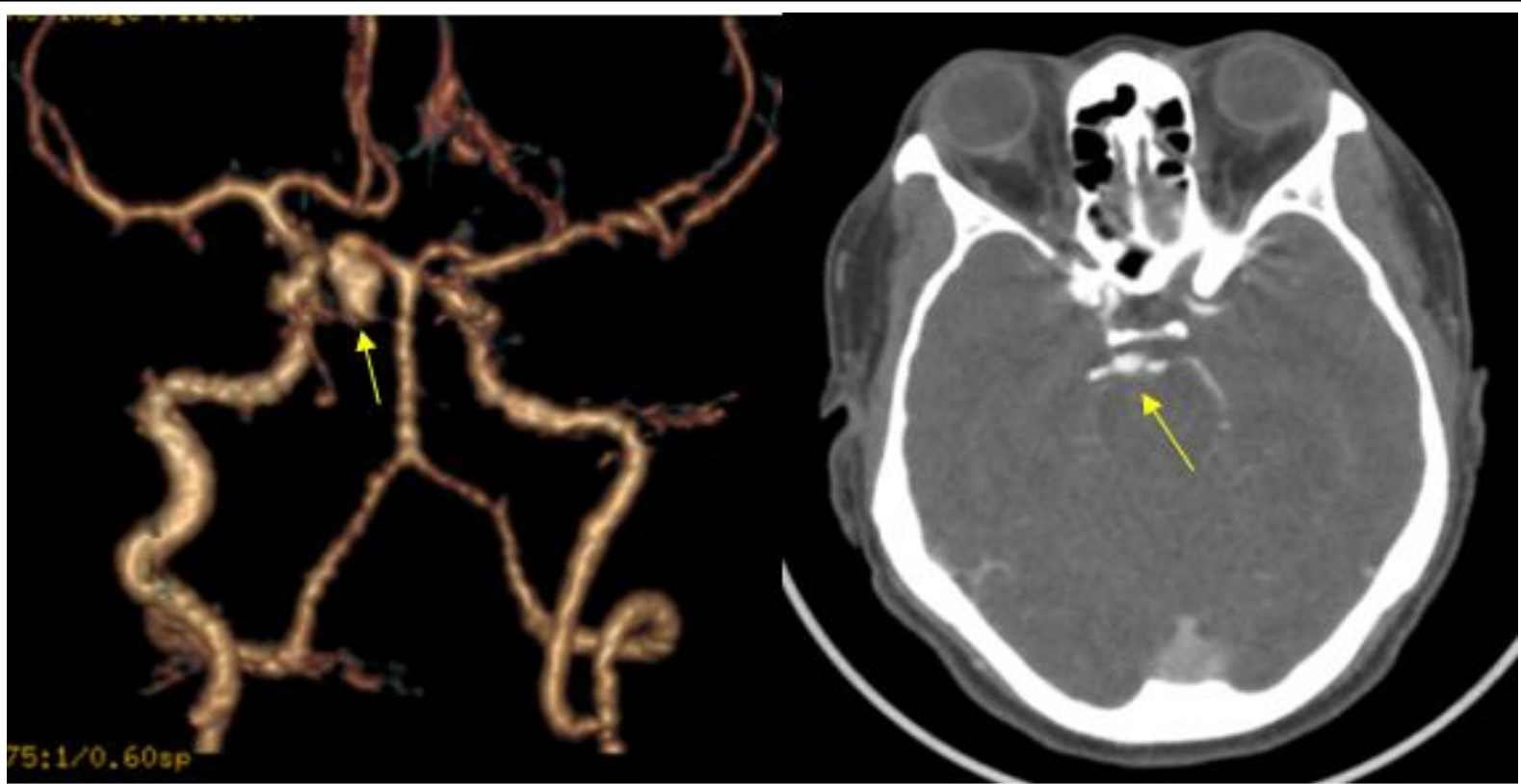

Fig.3: Volume rendering and axial CT angiography images of brain showing images of cerebral vessels with aneurysm involving the right PCA with aplastic left A1

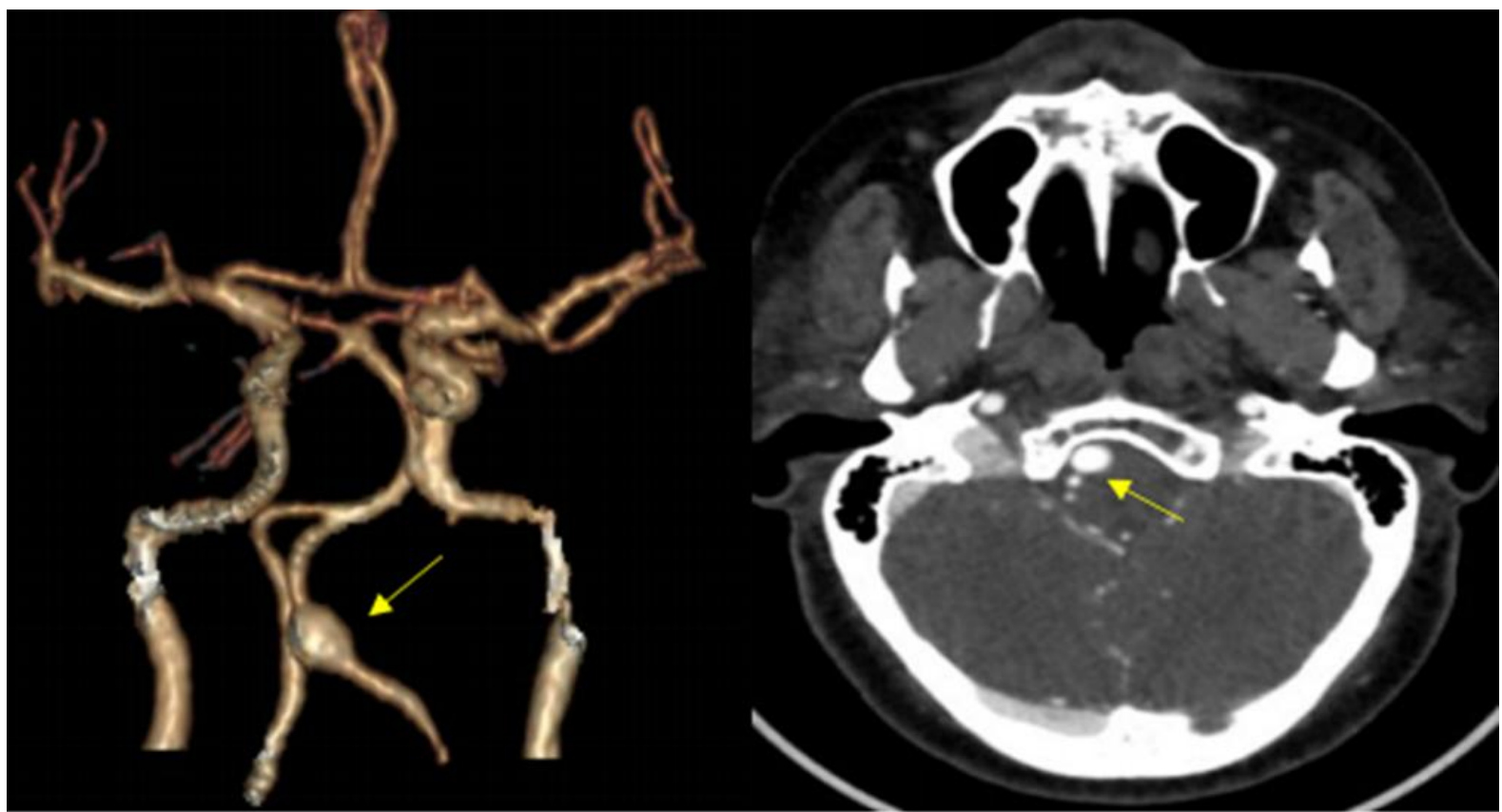

Fig. 4: Volume rendering and axial CT angiography images of the brain showing images of cerebral vessels with aneurysm involving the left vertebral artery

\section{Discussion}

Subarachnoid hemorrhage is most commonly caused due to the rupture of an intracranial aneurysm, head injury, cerebral arteriovenous malformation, arteriovenous fistula, etc. Aneurysmal rupture may also present with intraparenchymal hemorrhage (IPH), intraventricular hemorrhage (IVH), or subdural hemorrhage in $34 \%, 17 \%$ and $35 \%$ of cases, respectively ${ }^{1}$. Aneurysmal SAH is considered to be a medical emergency leading to death and permanent disability in nearly $50 \%$ of the survivors. It has a mortality rate ranging from 32$67 \%^{2}$. Risk factors for the development of intracranial aneurysms include both inherited and acquired causes [Table $1 \mathrm{a}, 1 \mathrm{~b}]^{3}$. 


Table 1a. Genetic conditions predisposing to aneurysm formation and
subarachnoid hemorrhage
Adult polycystic kidney disease
Coarctation of aorta
Marfan's syndrome
Hereditary hemorrhagic telangiectasia
Ehlers-Danlos syndrome
a1 antitrypsin deficiency

\begin{tabular}{|l|}
\hline Table 1 b. Acquired risk factors for intracranial aneurysms [2] \\
Increasing age \\
Hypertension \\
Smoking \\
Alcohol abuse \\
Estrogen deficiency \\
Hypercholesterolemia \\
Carotid artery stenosis \\
\hline
\end{tabular}

Jianfeng Liu et al. analyzed the relationship between demographic factors, medical history, aneurysmal features, and Fischer grades as a measure of hemorrhagic volume ${ }^{4}$. Patients with ruptured intracranial aneurysms present with the characteristic symptoms of "worst headache of life," nausea, vomiting, loss of consciousness, seizures, and neck stiffness ${ }^{5,6}$. The most crucial aspect that influences the volume of SAH is the site of the aneurysm, which predisposes them to hemorrhage directly into the parenchyma or ventricular system. CT Angiography, MR Angiography, and transcranial color-coded ultrasonography (TCCD) are the noninvasive techniques used in cases of ruptures intracranial aneurysms presenting with SAH. However, digital subtraction angiography remains the gold standard for the identification of the source of the bleed. High resolution multidetector computed tomography (CT) head scan is widely available and is considered as the primary investigation in patients with suspected SAH with a sensitivity of $95 \%$ on the day of hemorrhage ${ }^{7}$. It can demonstrate the source of the bleed and spaceoccupying lesions, causing mass effect such as obstructive hydrocephalus. Van Gijn J et al. stated that the sensitivity of the CT scanner to detect a SAH continues to decrease and is $50 \%$ sensitive after one week and $30 \%$ after two weeks of hemorrhage ${ }^{8}$. Intracranial aneurysms are saccular, dissecting, fusiform, serpentine, blood blister type, traumatic, mycotic, atheromatous, etc. of which, saccular noted to be the most common type. Based on the size, they were categorized as small $(<15 \mathrm{~mm})$, large $(15-25 \mathrm{~mm})$, giant (25$50 \mathrm{~mm})$ and supergiant $(>50 \mathrm{~mm})$. Most of the intracranial aneurysms encountered were small and subcategorized as small $(<5 \mathrm{~mm})$ and medium $(5-15 \mathrm{~mm})^{9}$. CT Angiography is a quick and noninvasive procedure that helps in the delineation of aneurysms and vascular spasm. It can depict the ruptured intracranial aneurysms with a pooled sensitivity and specificity of 98 and $100 \%$ respectively. Small aneurysms located near the skull base may be missed on a CT scan, which can be reduced by subtraction methods from enhanced to unenhanced data and selective bone removal, or "matched mask bone elimination." Brian JL et al. stated that $85 \%$ of the aneurysms were located around the anterior communicating artery (30-35\%), the posterior communicating artery (30-35\%), the middle cerebral artery bifurcation (20\%), the basilar artery $(5 \%)$ and the internal carotid artery (ICA 
)terminus or posterior wall ${ }^{10}$. This study comprises a total of 150 patients who have undergone NCCT for headache, seizures and suspected SAH at Great Eastern Medical School and Hospital from November 2016 to December 2018, of whom 20 patients were documented with ruptured intracranial aneurysms. Further imaging was done with computed tomography angiography (CTA). In this study, there is an equal incidence of SAH in males and females. The mean age of presentation was 51 years, with saccular being the most common type of aneurysm comprising of about $53.57 \%$. In our series, eight ICA and seven MCA aneurysms led to intraparenchymal hemorrhage into the adjacent temporal lobe. As the dome, which is the weakest portion of the aneurysm points laterally, it bled into the temporal lobe. Multiple aneurysms accounted for $25 \%$ in our study, predominantly on the left side and were associated with higher bleeding volumes. Specific aneurysmal sites are seen related to anatomical variations in the major intracranial vessels. Krzyzewski RM et al. stated aplasia/hypoplasia of the A1 segment of ACA is most commonly associated with aneurysm formation, comprising up to $36 \%$. According to Orakdogen $\mathrm{M}$ et al., arterial hypoplasia/aplasia were the most common vascular variants associated with aneurysms. Thorough knowledge of these variants is essential for proper treatment and to increase the success rate in the management of the patient. Endovascular coiling and surgical clipping are useful to treat large and symptomatic aneurysms. Small aneurysms $<7 \mathrm{~mm}$ are unlikely to rupture, but if rupture occurs, it leads to devastating consequences.

\section{Conclusion}

Any CT scan showing an intraparenchymal hemorrhage in the temporal lobe, with or without a frontal, parietal and intraventricular extension, should be looked in for any ruptured aneurysms even in the absence of SAH. Our study showed ruptured intracranial aneurysms with subarachnoid hemorrhage in $13 \%$ of the study population with no gender predilection. Left supraclinoid ICA was the most common vessel involved, and fetal PCA was the common anatomical variant associated with ruptured intracranial aneurysms.

\section{References}

1. Pasqualin A, Bazzan A, Cavazzani P, Scienza R, Licata C, Da Pian R: Intracranial hematomas following aneurysmal rupture: Experience with 309 cases. Surg Neurol

2. Yousef K, Crago E, Kuo CW, Horowitz M, Hravnak M. Predictors of delayed cerebral ischemia after aneurysmal hemorrhage: A cardiac focus. Neurocrit Care 2010;13:366-72.

3. Wagner $M$, Stenger $K$. Unruptured intracranial aneurysms: using evidence and outcomes to guide patient teaching. Crit Care Nurs Q 2005;28:341-54.

4. Jianfeng Liu, Jian Song, Di Zhao, Hui Li, Yingying $\mathrm{Lu}$, Guobiao $\mathrm{Wu}$, Kai Hou, Xuan Gao. Risk factors responsible for the volume of hemorrhage in aneurysmal subarachnoidhemorrhage. Neurology India 2016:64(4):686-91.

5. Liebenberg WA, Worth R, Firth GB, et al. Aneurysmal subarachnoid hemorrhage: guidance in making the correct diagnosis Postgrad Med J 2005;81:470-3.

6. Gorlick PB, Hier DB, Caplan LR, et al. Headache in acute cerebrovascular disease. Neurology 1986;36:1445-50.

7. der Wee N, Rinkel GJ, Hasan D, et al. Detection of subarachnoid hemorrhage on early CT: is lumbar puncture still needed after a negative scan? J Neurol Neurosurg Psychiatry 1995;58:357-9

8. Van Gijn J, van Dongen KJ: The time course of aneurysmal hemorrhage on computed tomograms. Neuroradiology 23:153-156, 1982.

9. Meyer PM, Schumacher HC, Higashida RT, et al. Reporting standards for 
endovascular repair of saccular intracranial cerebral aneurysms. Stroke 2009;40:e366e379.

10. Brisman JL, Song JK, Newell DW. Cerebral aneurysms. N Engl J Med 2006;355:928-939. 